\title{
Stage-dependent hierarchy of criteria in multiobjective multistage decision processes
}

\author{
Tadeusz Trzaskalik ${ }^{1, \dagger}$ \\ ${ }^{1}$ Faculty of Informatics and Communication, Department of Operations Research, \\ University of Economics in Katowice, ul. 1 Maja 50, 40-287 Katowice, Poland \\ E-mail: 〈tadeusz.trzaskalik@ue.katowice.pl〉
}

\begin{abstract}
This paper will consider a multiobjective, multistage discrete dynamic process with a changeable, state-dependent hierarchy of stage criteria determined by the decision maker. The goal of this paper is to answer the question of how to control a multistage process while taking into account both the tendency to achieve multiobjective optimization of the entire process and the time-varying hierarchy of stage criteria. We consider in detail possible situations, where the hierarchy of stage criteria changes over time in individual stages and is stage dependent. We present an interactive proposal to solving the problem, where the decision maker actively participates in finding the final realization of the process. The algorithm proposed is illustrated using a numerical example.
\end{abstract}

Keywords: MCDM, multiobjective multistage decision process, multiobjective dynamic programming, hierarchical problem

Received: November 28, 2016; accepted: January 17, 2017; available online: March 31, 2017

DOI: $10.17535 /$ crorr.2017.0001

\section{Introduction}

In this paper, we will deal with multistage decision processes. Decisions are made at the beginning of consecutive stages and evaluated using several evaluation criteria. Problems of this type are classified as multiobjective dynamic programmming problems. Among many and varied topics discussed at present there are many problems in which the hierarchization of evaluation criteria is an essential element. This is evident in the overview of outstanding papers in the field, presented below.

The paper [6] deals with motion generation in complex dynamical systems to achieve several concurrent objectives. Hierarchy of tasks and optimal control are two frameworks commonly used to this end. The first specifies control objectives as a number of quadratic functions to be minimized under strict priorities. The

${ }^{\dagger}$ Corresponding author 
second framework minimizes an arbitrary user-defined function of a future system state, thus considering its evolution over time.

The paper [5] presents a continuous-time contract whereby a top-level player can incentivize a hierarchy of players below him to act in his best interest, despite only observing the output of his direct subordinate. This paper extends Sannikov's approach from a situation involving asymmetric information between a principal and an agent to one of hierarchical information between several players. This paper identifies conditions subject to which dynamic programming construction of an optimal dynamic contract is reduced to a one-dimensional state space and one-dimensional control set, regardless of hierarchy size.

The paper [4] provides a rationale for central place theory based on dynamic programming formulation of a social planner's city hierarchy problem. The authors show that in any optimal solution there must be exactly only one immediate smaller city between two larger neighboring cities.

In the paper [1], the authors consider the bilevel knapsack problem (BKP), a hierarchical optimization problem with the feasible set determined by a set of optimal solutions for the parametric knapsack problem. They introduce a new reformulation of the BKP into a one-level integer programming problem using dynamic programming and subsequently propose an algorithm that solves the BKP in exactly two steps.

The paper [3] introduces a novel algorithm based on scan-line dynamic programmming. It uses the cost integration strategy for semi-global matching, a wellknown concept in stereo matching. The major novelty of the algorithm is that it embeds the scan-line dynamic programming approach into a hierarchical scheme, which allows it to handle large pixel displacements with an accuracy comparable to variational methods.

In the paper [2], the authors introduce the tree-like weighted set packing problem, which is a weighted set packing problem restricted to sets forming a tree-like hierarchical structure. They propose a dynamic programming algorithm with cubic time complexity.

The paper [7] develops a new algorithm based on a dynamic-programming strategy for constructing concept hierarchies from continuous attributes. The constructed trees have three merits: (1) they are global optimal trees, (2) each interval is partitioned into the most appropriate number of subintervals, and (3) the trees are balanced. Finally, the authors carry out an experimental study using real data to show its efficiency and effectiveness.

In the paper [16], a new hierarchical stereo algorithm is presented. The algorithm matches individual pixels in corresponding scanlines by minimizing a cost function. Several cost functions are compared. The algorithm achieves a tremendous gain in speed and memory requirements by implementing it hierarchically. The images are downsampled an optimal number of times and the disparity map of a lower level is used as an 'offset' disparity map at a higher level. 
In our paper, we consider multistage decision processes consisting of a finite number of stages determined by the decision maker. In evaluating of the admissible process realizations, we will use two types of criteria: stage criteria, which are related to the individual, fixed stages of the process, and multistage criteria, used to evaluate the overall realization of the process. We will consider the most frequently occurring situation, in which multistage criteria are sums of stage criteria.

In formulating the problem of process realization evaluation, we refer to the general notion of optimality in multiobjective problems [8]. We assume that the components of vector criteria functions are consecutive multistage criteria. As vector-optimal realizations we admit those that are non-dominated (in the criteria space) or efficient (in the decision space) [15].

Given all the considered criteria, both multistage and stage can be equally important for the decision maker. It does happen, however, that certain criteria be it stage of multistage - are more important than others, in which case the issue of criteria hierarchization occurs then naturally.

A change in importance of the criteria often influences decision making. Achieving a better stage evaluation of a criterion, which is important at a given stage, means that the DM often gives up on optimizing realization of multistage objectives. However, obtaining immediate profits may have a very significant negative impact on evaluating the entire process. For this reason, in the case of criteria hierarchization, it seems justified to focus on analyzing the values of both the stage and multistage criteria.

The problem of hierarchization of multistage and stage criteria has been discussed before. In [12], the problem of searching for the best process realization is discussed, specifically in the situation when a hierarchy of multistage criteria is given. Each time when the consecutive (with respect to importance) criteria were analyzed, the stage structure of the consecutive process realizations was analyzed. The changeability of hierarchies of stage criteria is also discussed in other papers. In [13], a hierarchy dependent on the joint value of stage criteria obtained in previous stages is discussed, while in [14], a hierarchy dependent on the current process state. These discussions continue in $[10,11]$, which also deals with group hierarchy. Changeable, weighted relevance of stage objectives are discussed in [9]. In each of these cases, the process realization that best satisfies the assumptions on hierarchization of stage and multistage criteria, is compared with a set of vector-optimal realizations.

The present paper attempts to answer the question of controlling a multistage process while taking into account both the tendency to multicriteria optimization of the entire process and the time-varying hierarchy of stage criteria. We will discuss in detail one of many possible situations, in which stage hierarchy varies in consecutive stages and depends on the actual stage. We will present an 
interactive proposal as a solution to this problem, in which the decision maker actively participates in finding the final realization of the process.

The present paper consists of five sections. In Section 2, we define the notation used and present the concept of vector optimization for a multiobjective decision process. In Section 3 we formulate the hierarchical problem discussed in the paper and propose a solution procedure. A detailed solution of an illustrative numerical example is found in Section 4. A summary completes the paper.

\section{Multistage, multiobjective discrete decision process}

We define $\overline{1, \mathrm{~T}}$ as the set of all integer numbers from 1 to $\mathrm{T}$ and denote it as follows:

$$
\overline{1, \mathrm{~T}}=\{1, \ldots, \mathrm{T}\}
$$

We consider a discrete decision process consisting of $\mathrm{T}$ stages. Let $\mathrm{y}_{\mathrm{t}}$ be the state variable at the beginning of stage number $t, Y_{t}$ - the set of all feasible state variables for stage $t, x_{t}$ - the decision variable for stage $t$ and $X\left(y_{t}\right)$ - the set of all feasible decision variables for stage $t$ and state $y_{t}$. We assume that all sets of states and decisions are finite. A stage realization is defined as follows:

$$
\mathrm{d}_{\mathrm{t}} \cong\left(\mathrm{y}_{\mathrm{t}}, \mathrm{x}_{\mathrm{t}}\right)
$$

Let $D_{t}$ be the set of all stage realizations in stage $t$.

We assume that for $\mathrm{t} \in \overline{1, \mathrm{~T}}$ the transformations

$$
\Omega_{\mathrm{t}}: \mathrm{D}_{\mathrm{t}} \rightarrow \mathrm{Y}_{\mathrm{t}+1}
$$

are given. A sequence of stage realizations $\mathrm{d}=\left(\mathrm{d}_{1}, \ldots, \mathrm{d}_{\mathrm{T}}\right)=\left(\mathrm{y}_{1}, \mathrm{x}_{1}, \mathrm{y}_{2}, \mathrm{x}_{2}, \ldots, \mathrm{y}_{\mathrm{T}}, \mathrm{x}_{\mathrm{T}}\right)$ is called a process realization, if

$$
\forall_{\mathrm{t} \in \overline{1, \mathrm{~T}}} \mathrm{y}_{\mathrm{t}+1}=\Omega_{\mathrm{t}}\left(\mathrm{y}_{\mathrm{t}}, \mathrm{x}_{\mathrm{t}}\right)
$$

Let $\mathrm{D}$ be the set of all the process realizations. We define

$$
\mathrm{D}_{\mathrm{t}}\left(\mathrm{y}_{\mathrm{t}}\right) \cong\left\{\left\{\left(\mathrm{y}_{\mathrm{t}}, \mathrm{x}_{\mathrm{t}}\right): \mathrm{x}_{\mathrm{t}} \in \mathrm{X}_{\mathrm{t}}\left(\mathrm{y}_{\mathrm{t}}\right)\right\}\right.
$$

to be the set of all period realizations of the process which begin in the given state $\mathrm{y}_{\mathrm{t}}$.

We consider $\mathrm{K}$ criteria and assume that stage criteria functions $F_{t}^{k}: \mathrm{D}_{\mathrm{t}} \rightarrow \mathrm{R}$ are defined for each stage $\mathrm{t}$ and for each $\mathrm{k}=1, \ldots, \mathrm{K}$,. For the given realization $\mathrm{d}$, we obtain the values 


$$
\begin{array}{rrrr}
\mathrm{F}_{1}^{1}\left(\mathrm{~d}_{1}\right) & \mathrm{F}_{1}^{2}\left(\mathrm{~d}_{1}\right) & \ldots & \mathrm{F}_{1}^{\mathrm{K}}\left(\mathrm{d}_{1}\right) \\
\ldots \ldots & \ldots \ldots \ldots \ldots \ldots \ldots \ldots \ldots \ldots \ldots \ldots \ldots \ldots \ldots \ldots \ldots \ldots \\
\mathrm{F}_{\mathrm{T}\left(\mathrm{d}_{\mathrm{T}}\right)}^{1} & \mathrm{~F}_{\mathrm{T}}^{2}\left(\mathrm{~d}_{\mathrm{T}}\right) & \ldots & \mathrm{F}_{\mathrm{T}}^{\mathrm{K}}\left(\mathrm{d}_{\mathrm{T}}\right)
\end{array}
$$

$\mathrm{F}$ is a vector-valued criterion function for evaluating the whole process and for $\mathrm{k}$ $=1, \ldots, \mathrm{K}$ its components $\mathrm{F}^{\mathrm{k}}$ are defined as follows:

$$
F^{k}(d)=\sum_{t=1}^{T} F_{k}^{T}\left(d_{t}\right)
$$

We postulate the maximization of all components of $\mathrm{F}$.

Let us assume that two process realizations: $\overline{\mathrm{d}}, \widetilde{\mathrm{d}}$ and vectors

$$
\begin{aligned}
& F(\bar{d}) \cong\left[F^{1}(\bar{d}), \ldots F^{K}(\bar{d})\right] \\
& F(\widetilde{d}) \cong\left[F^{K}(\widetilde{d}), \ldots F^{1}(\widetilde{d})\right]
\end{aligned}
$$

are given. The domination relation $\geq$ is defined as follows:

$$
\begin{gathered}
\mathrm{F}(\overline{\mathrm{d}}) \geq \mathrm{F}(\widetilde{\mathrm{d}}) \Leftrightarrow \\
\underset{\mathrm{k} \in 1, \mathrm{~K}}{\forall}\left[\mathrm{F}^{\mathrm{k}}(\overline{\mathrm{d}}) \geq \mathrm{F}^{\mathrm{k}}(\widetilde{\mathrm{d}})\right] \wedge \underset{\mathrm{i} \in \overline{1}, \mathrm{~K}}{\exists}\left[\mathrm{F}^{\mathrm{i}}(\overline{\mathrm{d}})>\mathrm{F}^{\mathrm{i}}(\widetilde{\mathrm{d}})\right]
\end{gathered}
$$

If $F(\overline{\mathrm{d}}) \geq F(\widetilde{\mathrm{d}})$ we say that vector $F(\overline{\mathrm{d}} d$ lominates vector $\quad F(\widetilde{\mathrm{d}})$ nd realization

$\overline{\mathrm{d}}$ is better than realization $\widetilde{\mathrm{d}}$. Realization ${ }^{*} \mathrm{~d}$ is said to be efficient if

$$
\left.\underset{\mathrm{d} \in \mathrm{D}}{\exists} \mathrm{F}(\overline{\mathrm{d}}) \geq \stackrel{*}{*}^{*}\right)
$$

Let ${ }^{*}$ denote the set of all efficient realizations for the given criterion function F. The problem of finding $\mathrm{D}$ is called the dynamic vector optimization problem. The set

$$
\dot{\mathrm{D}}(\overline{\mathrm{d}}) \cong\{\stackrel{*}{*} \stackrel{*}{\mathrm{D}}: \stackrel{*}{*}(\dot{\mathrm{d}}) \geq \mathrm{F}(\overline{\mathrm{d}})\}
$$

consists of all efficient realizations which are better than $\overline{\mathrm{d}}$. The algorithm for finding the set of all efficient realizations of the process and the algorithm for 
finding the set of efficient realizations better than the chosen one is described in $[15]$

\section{Description of the procedure}

Further on in the paper, we will address the situation when the hierarchy of stage criteria is important for the decision maker; this hierarchy was determined before the process started and does not undergo changes. However, making decisions concerning the individual stages requires realizing multistage objectives. The goal of the proposed interactive procedure is to make the decision maker aware of the consequences of stage decisions realizing multistage objectives, as well as pointing out new possibilities which result from analyzing stage objectives and multistage ones.

We assume that prior to the process starting, we need to plan what stage decisions are necessary. We start with a fixed hierarchy of stage criteria for all stages of the process. Establishing a hierarchy means placing the most important criterion at the first level, and the second most important criteria at the second level, and so on for the remaining levels. There is only one criterion at each hierarchy level.

Our assumption is a changeable hierarchy, that is, the importance of stage criteria differs across at least at two stages. This hierarchy can be determined by or imposed on the decision maker. At each stage, the most important criterion is distinguished. The hierarchy of the remaining (or some) criteria can be also determined, but is not necessary. Hence, at each stage, the set of stage criteria can be partitioned into two groups, one with a determined hierarchy and the other with no determined hierarchy. The former always has at least one element, the latter can be an empty set. We regard all non-hierarchical criteria as equally important; they are also less important than the hierarchical ones.

We need to solve the problem of selecting the initial state for the first stage, that is, the state at which process realization begins. The first proposed solution is to take this initial state of the first stage for which the value of the stage realization starting in this state is largest. This is so because the decision maker's immediate objective is to maximize the values of the stage criteria. If there is more than one such state, we can choose any of them, and the consecutive states will be considered when the procedure is repeated (if necessary).

Since the decision maker, when making a stage decision, can take into account also those stage realizations for which the stage values of the most important criterion at the first stage are close to the optimal value, we make another suggestion for the selection of the initial stage. Namely, we suggest that the decision maker determine the approximate number $M$ of necessary realizations. Next, for each state, we find the best realization and M-1 subsequent realizations and sum up the obtained values. As the initial state, we select the one for which this sum is the largest. 
The decision maker may be interested both in those stage realizations which allow to obtain maximal values of the currently considered stage criteria, and those that are almost optimal and fall within the determined tolerance intervals. Such decisions are called decisions satisfactory as regards the relevant stage criteria.

The problem is solved in consecutive stages, starting with the first stage. At each subsequent stage, the hierarchized stage criteria are considered consecutively, starting with the most important criterion.

When considering the most important stage criterion, we take into account all the stage decisions admissible in the current process state. The decision maker provides an initial tolerance interval for the maximal value of the current stage criterion. Accordingly, we determine the initial set of satisfactory realizations. Of course, the cardinality of this set depends on the extent to which the decision maker is willing to relinquish the optimal value. For this reason, if the tolerance interval determined by the decision maker is too narrow, we suggest extending it. The decision maker then agrees to lower the requirements for the respective criterion. On the other hand, if the cardinality of the realization set is too large, the decision maker can narrow the suggested tolerance interval, which guarantees a better value of the criterion in the final solution. Once the decision maker accepts the tolerance interval, we obtain a set of satisfactory realizations for the respective criterion. Then the next most important stage criterion (if it exists) can be considered; here we take into account only those realizations which were satisfactory for the previously considered stage criteria.

Having considered all hierarchized criteria from consecutive hierarchy levels, the decision maker selects the final stage decision from the last set of satisfactory stage realizations. Helpful in this selection can be the value of the index which describes the joint relative change of the value of the given realization with respect to possible maximal changes of the individual stage criteria. A detailed method of constructing this index is found in the description of the algorithm.

Having identified the stage decision, we use the transformation function and determine the initial state for the next stage.

This procedure is repeated for all consecutive stages and the final stage. The result is a process realization satisfactory for all hierarchized stage criteria, or satisfactory realization for short. This realization is added to the set of potential realizations, and is the basis for the decision maker's final selection.

We next analyze the values of multistage criteria for a satisfactory realization. Using the efficiency testing procedure, we check whether this realization is efficient or not. If not, we generate efficient realizations better than this one and add them to the set of potential realizations. Thus, the set of potential realizations contains a satisfactory realization and efficient realizations better than the satisfactory one (if they exist).

When performing a preliminary analysis of the set of potential realizations, the decision maker may accept the set as sufficient for the final decision (which 
consists in indicating one of the potential realizations as the final realization), or else extend this set (by repeating the entire procedure and selecting a state not yet considered as the initial state).

Next, the decision maker (jointly with an analyst) uses expert knowledge to analyze in detail the values of the stage and multistage criteria of all the generated potential realizations. As a result, the decision maker can select a satisfactory and efficient realization (if it exists, of course) as the final decision; he/she can also select a satisfactory realization which is not efficient or else an efficient realization which is not satisfactory.

The decision maker can also - in extending the set of potential realizations repeat the entire procedure once again, starting with the state not previously considered as the initial state. If the decision maker does not accept any of these possibilities, he/she can give up on making a decision using the above procedure.

\section{The algorithm}

Step 1. The decision maker determines a hierarchy of stage criteria for each stage. We determine the values $\mathrm{K}(1), \ldots, \mathrm{K}(\mathrm{T})$ which describe the number of hierarchized criteria in stages $t \in \overline{1, T}$. The stage hierarchy is described by the sets of criteria numbers $K_{\mathrm{t}}=\left\{\mathrm{k}_{\mathrm{t}}{ }^{1}, \ldots, \mathrm{k}_{\mathrm{t}}^{\mathrm{K}(\mathrm{t})}\right\}, \mathrm{t} \in \overline{1, \mathrm{~T}}$. Each of them contains numbers of stage criteria, sorted from the most important to the least important criterion. ${ }^{+}$

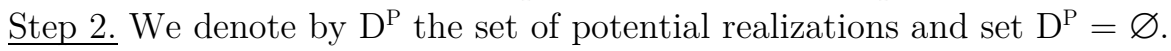
Step 3. We denote the most important stage criterion in stage 1 by $\mathrm{F}_{1}{ }^{1}$. We select the initial state using one of the following possibilities:

a) The set $Y_{1}$ of states for Stage 1 is finite and consists of $N$ elements which can be written as the following sequence:

$$
\mathrm{Y}_{1}=\left\{\mathrm{y}_{1}^{(1)}, \mathrm{y}_{1}^{(2)}, \ldots, \mathrm{y}_{1}^{(\mathrm{N})}\right\}
$$

For the consecutive states from set $\mathrm{Y}_{1}$ we find the maximal stage values using the formula:

for $\mathrm{n}=1, \ldots, \mathrm{N}$.

$$
F_{1}^{l^{*}}\left(y_{1}^{(n)}\right)=\max _{x_{1} \in X_{1}\left(y_{1}^{(n)}\right)} F_{1}^{l}\left(y_{1}^{(n)}, x_{1}\right)
$$

We look for state $\mathrm{y}_{1}{ }^{(\mathrm{s})}$ for which the value $\mathrm{F}_{1}{ }^{*}\left(\mathrm{y}_{1}{ }^{(\mathrm{n})}\right)$ is maximal. We find state $\mathrm{y}_{1}^{(\mathrm{s})}$ as follows:

$$
\mathrm{y}_{1}{ }^{(\mathrm{s})}=\arg \max \left\{\mathrm{F}_{1}{ }^{1^{*}}\left(\mathrm{y}_{1}{ }^{(1)}\right), \ldots, \mathrm{F}_{1}{ }^{1^{*}}\left(\mathrm{y}_{1}{ }^{(\mathrm{N})}\right)\right\}
$$

\footnotetext{
$\$$ For instance, when at stage 3 in a set with four stage criteria, the most important criterion is $\mathrm{F}_{3}{ }^{2}$, the second most important is $\mathrm{F}_{3}{ }^{4}$, and the remaining criteria are not hierarchized, we then have $\mathrm{K}_{3}=\{2,4\}$ and $\mathrm{K}_{3}=2$.
} 
b) We ask the decision maker to give the probable number $\mathrm{M}$ of realizations which will be optimal or near optimal with respect to the criterion considered first in stage 1 . Let $\max _{\mathrm{m}}$ be the $m$-th consecutive maximal value. For $\mathrm{n}=1, \ldots, \mathrm{N}$ and the consecutive states $\mathrm{y}^{(\mathrm{n})}$ of the process, we calculate $\mathrm{M}$ near optimal values as

$$
\max _{x_{1} \in X_{1}\left(y_{1}^{(n)}\right)} F_{1}\left(y^{(n)}, x_{1}\right), \max _{x_{1} \in X_{1}\left(y_{1}^{(n)}\right)} F_{1}\left(y^{(n)}, x_{1}\right), \ldots, \max _{x_{1} \in X_{1}\left(y_{1}^{(n)}\right)} F_{1}\left(y^{(n)}, x_{1}\right)
$$

and their average. We search for state $\mathrm{y}_{1}{ }^{(\mathrm{s})}$ for which this value is maximal:

$$
y_{1}^{(s)}=\arg \frac{\sum_{m=1}^{M} \max _{x_{1} \in X_{1}\left(y^{(n)}\right)} F_{1}\left(y^{(n)}, x_{1}\right)}{M}
$$

c) If the decision maker does not accept any of the two above suggestions, we ask $\mathrm{him} /$ her to indicate the preferred initial state.

$\underline{\text { Step } 4}$. We start the procedure with Stage 1 and hence set $t=1$.

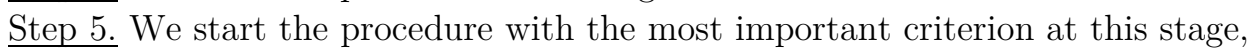
and hence set $\mathrm{i}=1$.

Step 6. We consider the $k$ th criterion $\left(k=k_{t}{ }^{i}\right)$. We find the maximal value $\mathrm{F}_{\mathrm{t}} \mathrm{k}^{*}$ admitted by criterion $\mathrm{F}_{\mathrm{t}}{ }^{\mathrm{k}}$ for admissible process realizations starting at the respective state.

$\underline{\text { Step } 7 .}$ We inform the decision maker of the value $\mathrm{F}_{\mathrm{t}}{ }^{\mathrm{k}}$ and ask him/her to supply the value of $\varepsilon_{t}{ }^{k}$, so as to determine the tolerance interval $\left[\mathrm{F}_{\mathrm{t}}{ }^{\mathrm{k} *}-\varepsilon_{\mathrm{t}}{ }^{\mathrm{k}}, \mathrm{F}_{\mathrm{t}}^{\mathrm{k} *}\right]$.

Step 8. We determine all the stage realizations for criterion $F_{\mathrm{ik}}$ with values in the interval $\left[\mathrm{F}_{\mathrm{t}}^{\mathrm{k} *}-\varepsilon_{\mathrm{t}}^{\mathrm{k}}, \mathrm{F}_{\mathrm{t}}^{\mathrm{k} *}\right]$. We denote this set by $\mathrm{D}_{\mathrm{t}}^{\mathrm{k}}$.

Step 9. The decision maker is informed of the obtained cardinality of the set and is then asked to accept the result. If the decision maker accepts this cardinality, we proceed to Step 12 .

Step 10. If the decision maker finds this cardinality too large, we ask him/her to give a new tolerance level or to indicate what cardinality is appropriate in his/her opinion. We delete the superfluous realizations and return to Step 8.

Step 11. If the decision maker finds this cardinality too small, we ask him/her to give a new tolerance level or indicate the number of stage realizations to be added. We return to Step 8.

$\underline{\text { Step } 12}$. We set $\mathrm{i}=\mathrm{i}+1$ and check whether $\mathrm{i} \leq \mathrm{K}(\mathrm{t})$. If so, we proceed to Step 6.

Step 13. We select the preferred stage realization from the reduced set $\mathrm{D}_{\mathrm{t}}$ ' of realizations as described above, and check whether this set contains dominated stage realizations; if so, we delete them. After that, set $\mathrm{D}_{\mathrm{t}}$ ' consists of $\mathrm{P}^{\prime}$ elements. 
For each stage realization $\mathrm{d}_{\mathrm{t}}{ }^{(\mathrm{p})} \in \mathrm{D}_{\mathrm{t}}$ ' we calculate the index $\mathrm{f}_{\mathrm{pk}}$ for the consecutive criteria by dividing the value $\mathrm{F}_{\mathrm{t}}{ }^{\mathrm{k}}\left(\mathrm{d}_{\mathrm{t}}{ }^{(\mathrm{p})}\right)$ by the maximal achievable value of stage criterion $\mathrm{F}_{\mathrm{t}}{ }^{\mathrm{k}}$ in $\mathrm{D}^{\prime}$. We thus obtain:

$$
f_{p k}=\frac{F_{t}^{k}\left(d_{t}^{(p)}\right)}{\max _{d \in D^{\prime}} F^{k}\left(d_{t}\right)}
$$

A P' $\times \mathrm{K}$ matrix $F=\left[\mathrm{f}_{\mathrm{pk}}\right]$ is formed from these values. As the stage decision, we suggest selecting a decision number $\mathrm{p}^{\circ}$, for which the sum of the elements in the corresponding row of matrix $\mathrm{F}$ is the largest, that is

$$
p^{0}=\arg \max \sum_{k=1}^{K} f_{p k}
$$

If the decision maker does not accept this suggestion, he/she performs the selection autonomously, by analyzing the values of matrix $F$.

Step 14. Using the transformation, we determine the process state at the end of the stage; this is at the same time the initial state of the next stage or the final state of the process. We then have:

$$
\mathrm{y}_{\mathrm{t}+1}=\Omega_{\mathrm{t}}\left(\mathrm{y}_{\mathrm{t}}, \mathrm{x}_{\mathrm{t}}\right)
$$

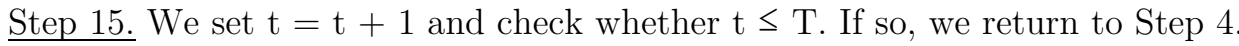

Step 16. We add the realization $\mathrm{d}^{\mathrm{o}}$ to set $\mathrm{D}^{\mathrm{P}}$ of potential realizations:

$$
\left(\mathrm{D}^{\mathrm{P}}=\mathrm{D}^{\mathrm{P}} \cup\left\{\mathrm{d}^{\circ}\right\}\right.
$$

Step 17. Using the algorithm for verifying efficiency, we check whether the realization generated is efficient. If not, we generate a better set $\mathrm{D}^{*}\left(\mathrm{y}^{*}\right)$ of efficient realizations than the obtained realization.

Step 18. We add the realizations from set $\mathrm{D}^{*}\left(\mathrm{y}^{*}\right)$ to set $\mathrm{D}^{\mathrm{P}}$ of potential realizations:

$$
\mathrm{D}^{\mathrm{P}:}=\mathrm{D}^{\mathrm{P}} \cup \mathrm{D}^{*}\left(\mathrm{y}^{*}\right)
$$

Step 19. We ask the decision maker whether to repeat the procedure to obtain the next satisfactory realization. If not, we proceed to Step 20.

Step 20. The decision maker is asked to indicate a state not yet considered, as the next initial state. We go to Step 4.

Step 21. The decision maker, using his/her expert knowledge, analyzes the set of potential decisions, while taking into account the stage hierarchy and the values of stage and multistage criteria. As a result, the decision maker:

a) Indicates one of the potential realizations as the final realization. 
b) Repeats the procedure starting with Step 2, to obtain a new potential realization.

c) Eliminates some of the realizations previously obtained from the set of potential realizations.

d) Changes the stage hierarchy and repeats the entire procedure.

e) Gives up making the decision using the procedure described above.

\section{Numerical illustration}

We now consider a two-stage, two-criteria decision process in which the transformation function has the form:

$$
\mathrm{y}_{\mathrm{t}+1}{ }^{(\mathrm{j})}=\Omega_{\mathrm{t}}\left(\mathrm{y}_{\mathrm{t}}{ }^{(\mathrm{i})}, \mathrm{x}_{\mathrm{t}}{ }^{(\mathrm{j})}\right)=\mathrm{x}_{\mathrm{t}}{ }^{(\mathrm{j})}
$$

that is, the decision consists in the selection of the next stage.

We denote stage realization $\mathrm{d}_{t}{ }^{\mathrm{ij}}$ which comprises state $\mathrm{y}_{t}^{(\mathrm{i})}$ and decision $\mathrm{x}_{\mathrm{t}}{ }^{(\mathrm{j})}$ as follows:

$$
\mathrm{d}_{\mathrm{t}}^{\mathrm{ij}}=\left(\mathrm{y}_{\mathrm{t}}{ }^{(\mathrm{i})}, \mathrm{x}_{\mathrm{t}}{ }^{(\mathrm{j})}\right)
$$

The values of the stage criteria, the same in both stages, are shown in Tables 1 and 2 .

\begin{tabular}{|c|c|c|c|c|c|c|c|c|c|c|c|}
\hline & Decision & 0 & 1 & 2 & 3 & 4 & 5 & 6 & 7 & 8 & 9 \\
\hline State & 0 & 420 & 451 & 433 & 494 & 462 & 400 & 455 & 459 & 438 & 452 \\
\cline { 2 - 11 } & 1 & 443 & 417 & 499 & 429 & 486 & 498 & 438 & 494 & 424 & 436 \\
\cline { 2 - 11 } & 2 & 429 & 490 & 491 & 434 & 494 & 484 & 420 & 480 & 458 & 482 \\
\hline 3 & 430 & 489 & 413 & 492 & 488 & 434 & 487 & 423 & 482 & 496 \\
\hline 4 & 414 & 407 & 418 & 409 & 460 & 456 & 454 & 452 & 419 & 446 \\
\hline 5 & 454 & 489 & 409 & 454 & 416 & 413 & 439 & 441 & 434 & 492 \\
\hline & 4 & 455 & 462 & 427 & 483 & 460 & 437 & 456 & 493 & 468 & 436 \\
\hline 7 & 438 & 439 & 494 & 449 & 446 & 422 & 491 & 437 & 425 & 455 \\
\hline & 8 & 490 & 418 & 449 & 410 & 429 & 454 & 439 & 422 & 434 & 438 \\
\hline & 9 & 437 & 424 & 447 & 497 & 433 & 480 & 488 & 464 & 406 & 492 \\
\hline
\end{tabular}

Table 1: Values of stage criteria $F_{t}^{1}(t=1,2)$ 


\begin{tabular}{|c|c|c|c|c|c|c|c|c|c|c|c|}
\hline & Decision & 0 & 1 & 2 & 3 & 4 & 5 & 6 & 7 & 8 & 9 \\
\hline State & 0 & 69 & 66 & 69 & 59 & 54 & 64 & 55 & 63 & 58 & 60 \\
\cline { 2 - 11 } & 1 & 59 & 55 & 63 & 62 & 53 & 67 & 61 & 65 & 62 & 69 \\
\cline { 2 - 11 } & 2 & 57 & 63 & 65 & 54 & 55 & 52 & 56 & 59 & 69 & 61 \\
\cline { 2 - 11 } & 3 & 52 & 67 & 61 & 61 & 69 & 59 & 65 & 51 & 52 & 69 \\
\cline { 2 - 11 } & 4 & 68 & 52 & 64 & 56 & 56 & 62 & 67 & 66 & 67 & 61 \\
\hline & 5 & 53 & 65 & 69 & 63 & 68 & 50 & 50 & 58 & 64 & 54 \\
\hline & 6 & 58 & 58 & 65 & 52 & 69 & 61 & 57 & 54 & 56 & 57 \\
\cline { 2 - 11 } & 7 & 51 & 56 & 63 & 58 & 52 & 53 & 52 & 60 & 53 & 62 \\
\cline { 2 - 11 } & 8 & 52 & 58 & 69 & 51 & 50 & 50 & 56 & 51 & 55 & 54 \\
\cline { 2 - 11 } & 9 & 60 & 63 & 60 & 52 & 51 & 53 & 69 & 59 & 63 & 53 \\
\hline
\end{tabular}

Table 2: Values of stage criteria $F_{t}^{2}(t=1,2)$

Below is an application of the proposed procedure.

\section{Stage 1}

Step 1. The decision maker has determined that the most important criterion at this stage is $F_{1}{ }^{1}$, and criterion $F_{1}{ }^{2}$ in the second stage. We have $\mathrm{K}(1)=\mathrm{K}(2)=2$ and $K_{1}=\{1,2\}, K_{2}=\{2,1\}$.

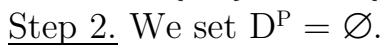

Step 3. We select the initial state using suggestion b). We assume that there will probably be four realizations optimal or near optimal with respect to criterion $\mathrm{F}_{1}{ }^{1}$ to be considered in Stage 1. We then calculate the following:

$$
\begin{aligned}
& \frac{\sum_{m=1}^{4} \max _{M} F_{1}^{1}\left(y^{(0)}, x_{1}\right)}{4}=467,5 \\
& \frac{\sum_{m=1}^{4} \max _{M} F_{1}^{1}\left(y^{(5)}, x_{1}\right)}{4}=472,5 \\
& \frac{\sum_{m=1}^{4} \max _{M} F_{1}^{1}\left(y^{(1)}, x_{1}\right)}{4}=494,25 \\
& \frac{\sum_{m=1}^{4} \max _{M} F_{1}^{1}\left(y^{(6)}, x_{1}\right)}{4}=476,5
\end{aligned}
$$




$$
\begin{gathered}
\frac{\sum_{m=1}^{4} \max _{M} F_{1}^{1}\left(y^{(2)}, x_{1}\right)}{4}=489,75 \quad \frac{\sum_{m=1}^{4} \max _{M} F_{1}^{1}\left(y^{(7)}, x_{1}\right)}{4}=472,25 \\
\frac{\sum_{m=1}^{4} \max _{M} F_{1}^{1}\left(y^{(3)}, x_{1}\right)}{4}=491,25 \\
\frac{\sum_{m=1}^{4} \max _{M} F_{1}^{1}\left(y^{(8)}, x_{1}\right)}{4}=457,75 \\
\frac{\sum_{m=1}^{4} \max _{M} F_{1}^{1}\left(y^{(4)}, x_{1}\right)}{4}=455,5 \\
\frac{\sum_{m=1}^{4} \max _{M} F_{1}^{1}\left(y^{(9)}, x_{1}\right)}{4}=489,25
\end{gathered}
$$

According to formula (12), the initial state is $\mathrm{y}_{1}{ }^{(1)}$.

Step 4. We set $\mathrm{t}=1$.

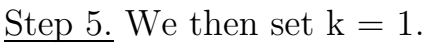

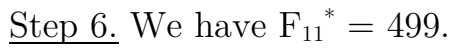

Step 7. We inform the decision maker of the value $\mathrm{F}_{1^{1}}{ }^{*}$. The decision maker is willing to lower the requirements for the first stage criterion by $5 \%$, that is $\varepsilon_{1}{ }^{1}=$ 24.95. Therefore, the tolerance interval is [474.05; 499].

Step 8. We determine all the stage realizations for criterion $\mathrm{F}_{11}$, where the criterion values lie in the interval $[474.05 ; 499]$. These are the realizations: $\mathrm{d}_{1}{ }^{12}, \mathrm{~d}_{1}{ }^{14}, \mathrm{~d}_{1}{ }^{15}$ and $d_{1}{ }^{17}$. We find the stage values of the first criterion:

$$
\mathrm{F}_{1}\left(\mathrm{~d}_{1}{ }^{12}\right)=499 \quad \mathrm{~F}_{1}\left(\mathrm{~d}_{1}{ }^{14}\right)=486 \quad \mathrm{~F}_{1}\left(\mathrm{~d}_{1}{ }^{15}\right)=498 \quad \mathrm{~F}_{1}\left(\mathrm{~d}_{1}{ }^{17}\right)=494
$$

Step 9. We inform the decision maker that set $\mathrm{D}_{1}{ }^{1}$ ' has four elements. The decision maker accepts the result. We reduce the set of acceptable decisions. We then have:

$$
\mathrm{D}_{1}{ }^{1}=\left\{\mathrm{d}_{1}{ }^{12}, \mathrm{~d}_{1}{ }^{14}, \mathrm{~d}_{1}{ }^{15}, \mathrm{~d}_{1}{ }^{17}\right\}
$$

We proceed to Step 12 .

$\underline{\text { Step } 12}$. We set $\mathrm{i}=2$. Since $\mathrm{i} \leq \mathrm{K}(1)=2$, we proceed to Step 6 .

Step 6. We have $\mathrm{F}_{1}{ }^{*}=69$.

Step 7. We inform the decision maker about the value $\mathrm{F}_{1^{2}}$. The decision maker is willing to lower the requirements for the second stage criterion by $10 \%$, that is, $\varepsilon_{1}^{2}=6.9$, and we obtain the tolerance interval $[62.1 ; 69]$. 
Step 8. We determine all the stage realizations from set $\mathrm{D}_{1}{ }^{1}$, for criterion $\mathrm{F}_{12}$, where the criterion values fall within the tolerance interval $[62.1 ; 69]$. These are the following realizations: $\mathrm{d}_{1}{ }^{12}, \mathrm{~d}_{1}{ }^{15}$ and $\mathrm{d}_{1}{ }^{17}$. For these realizations, we read the stage values of criterion 2 , and get:

$$
\mathrm{F}_{1}^{2}\left(\mathrm{~d}_{1}{ }^{12}\right)=63 \quad \mathrm{~F}_{1}{ }^{2}\left(\mathrm{~d}_{1}{ }^{15}\right)=67 \quad \mathrm{~F}_{1}{ }^{2}\left(\mathrm{~d}_{1}{ }^{17}\right)=65
$$

Step 9. We inform the decision maker that $\mathrm{D}_{1}{ }^{2}$ ' has three elements. The decision maker accepts this cardinality. We proceed to Step 12.

$\underline{\text { Step } 12}$. We set $\mathrm{i}=3$ and have $\mathrm{i}>\mathrm{K}(1)=2$.

Step 13. We select a satisfactory stage realization for Stage 1. The values of the stage criteria for the three stage realizations from set $\mathrm{D}_{1}{ }^{2}$, now considered are the following:

$$
\begin{array}{ll}
\mathrm{F}_{1}\left(\mathrm{~d}_{1}{ }^{12}\right)=499 & \mathrm{~F}_{1}{ }^{2}\left(\mathrm{~d}_{1}{ }^{12}\right)=63 \\
\mathrm{~F}_{1}\left(\mathrm{~d}_{1}{ }^{15}\right)=498 & \mathrm{~F}_{1}{ }^{2}\left(\mathrm{~d}_{1}{ }^{15}\right)=67 \\
\mathrm{~F}_{1}\left(\mathrm{~d}_{1}{ }^{17}\right)=494 & \mathrm{~F}_{1}{ }^{2}\left(\mathrm{~d}_{1}{ }^{17}\right)=65
\end{array}
$$

Stage realization $d_{1}{ }^{17}$ is dominated, hence it is removed from further considerations. We form matrix $\mathrm{F}$ :

$$
\left[\begin{array}{ll}
499 / 499 & 63 / 69 \\
498 / 499 & 67 / 69
\end{array}\right]=\left[\begin{array}{cc}
1 & 0.913043 \\
0.997996 & 0.971014
\end{array}\right]
$$

The realization index for $\mathrm{d}_{1}{ }^{12}$ is 1.913043 , while for $\mathrm{d}_{1}{ }^{15}$ it is 1.96901 . We select $\mathrm{d}_{1}{ }^{15}$ as the stage realization.

Step 14. Using the transfer function we obtain: $\Omega_{1}\left(\mathrm{~d}_{1}{ }^{15}\right)=\mathrm{y}_{2}{ }^{(5)}$.

Step 15. We set $\mathrm{t}=2$ and return to Step 5 .

Stage 2.

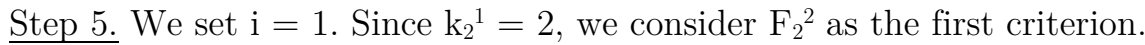

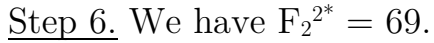

Step 7. We inform the decision maker of the value $\mathrm{F}_{1}{ }^{2 *}$. The decision maker is willing to lower the requirements for stage criterion $\mathrm{F}_{1}{ }^{2}$ by $10 \%$, that is, $\varepsilon_{2}{ }^{2}=6.9$, and we obtain the tolerance interval $[62.1 ; 69]$.

Step 8. We determine all the stage realizations for which the values of criterion $\mathrm{F}_{2}{ }^{2}$ are within the interval $[62.1 ; 69]$. These are: $\mathrm{d}_{2}{ }^{51}, \mathrm{~d}_{2}{ }^{52}, \mathrm{~d}_{2}{ }^{53}, \mathrm{~d}_{2}{ }^{54}$ and $\mathrm{d}_{2}{ }^{58}$. We find stage values of $\mathrm{F}_{2}^{2}$ for these realizations. We then have:

$$
\mathrm{F}_{2}\left(\mathrm{~d}_{2}{ }^{51}\right)=65, \mathrm{~F}_{2}\left(\mathrm{~d}_{2}{ }^{52}\right)=69, \mathrm{~F}_{2}\left(\mathrm{~d}_{2}{ }^{53}\right)=63, \quad \mathrm{~F}_{2}\left(\mathrm{~d}_{2}{ }^{54}\right)=68, \mathrm{~F}_{2}\left(\mathrm{~d}_{2}{ }^{58}\right)=64
$$

Step 9. We inform the decision maker that the obtained set has five elements. The decision maker decides that the cardinality of $\mathrm{D}_{2}{ }^{2}$ ' based on the current 
tolerance threshold is too large and gives a new tolerance threshold $\varepsilon_{2}{ }^{2}=4$. We return to Step 8.

Step 8. We determine all the stage realizations, for which the values of criterion $\mathrm{F}_{2}{ }^{2}$ lie within the interval [65; 69]. These are: $\mathrm{d}_{2}{ }^{51}, \mathrm{~d}_{2}{ }^{52}, \mathrm{~d}_{2}{ }^{54}$ and $\mathrm{d}_{2}{ }^{58}$. We find stage values of $\mathrm{F}_{2}^{2}$ for them. We have:

$$
\mathrm{F}_{2}\left(\mathrm{~d}_{2}{ }^{51}\right)=65 \quad \mathrm{~F}_{2}\left(\mathrm{~d}_{2}{ }^{52}\right)=69 \quad \mathrm{~F}_{2}\left(\mathrm{~d}_{2}{ }^{54}\right)=68 \quad \mathrm{~F}_{2}\left(\mathrm{~d}_{2}{ }^{58}\right)=64
$$

Step 9. We inform the decision maker that the obtained set has four elements. The decision maker accepts the result. We have:

$$
\mathrm{D}_{2}{ }^{2}=\left\{\mathrm{d}_{2}{ }^{51}, \mathrm{~d}_{2}{ }^{52}, \mathrm{~d}_{2}{ }^{54}, \mathrm{~d}_{2}{ }^{58}\right\}
$$

We go to Step 12.

Step 12. We set $\mathrm{i}=2$. Since $\mathrm{i} \leq \mathrm{K}(2)=2$, we return to Step 6 .

Step 6. We have $\mathrm{F}_{2}{ }^{{ }^{*}}=489$.

Step 7. We inform the decision maker of the value $\mathrm{F}_{2}{ }^{1 *}$. The decision maker is willing to lower the requirements for stage criterion $\mathrm{F}_{2}{ }^{1}$ by $\varepsilon_{2}{ }^{1}=60$, and we obtain the tolerance interval $[429 ; 489]$.

Step 8. We determine all stage realizations from set $\mathrm{D}_{2}{ }^{2}$, for criterion $\mathrm{F}_{2}{ }^{1}$, with the criterion values falling within the tolerance interval [429;489]. These are $\mathrm{d}_{2}{ }^{51}$ and $\mathrm{d}_{2}{ }^{58}$. We read the stage values of criterion $\mathrm{F}_{2}{ }^{1}$ for them. We have:

$$
\mathrm{F}_{2}{ }^{1}\left(\mathrm{~d}_{2}{ }^{51}\right)=489 \quad \mathrm{~F}_{2}{ }^{1}\left(\mathrm{~d}_{2}{ }^{58}\right)=434
$$

Step 9. We inform the decision maker that set $\mathrm{D}_{2}{ }^{1}$ ' has two elements. The decision maker accepts the cardinality of the set.

We go to step 12 .

$$
\mathrm{D}_{2}{ }^{1}=\left\{\mathrm{d}_{2}{ }^{51}, \mathrm{~d}_{2}{ }^{58}\right\}
$$

$\underline{\text { Step } 12}$. We set $\mathrm{i}=3$. We have $\mathrm{i}>\mathrm{K}(2)=2$.

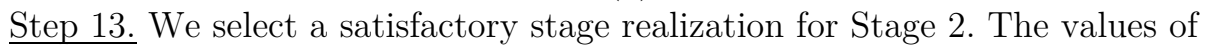
stage criteria for the two stage realizations from set $\mathrm{D}_{2}{ }^{1}$, are as follows:

$$
\begin{array}{ll}
\mathrm{F}_{2}{ }^{1}\left(\mathrm{~d}_{2}{ }^{51}\right)=489 & \mathrm{~F}_{2}{ }^{2}\left(\mathrm{~d}_{2}{ }^{51}\right)=65 \\
\mathrm{~F}_{2}{ }^{1}\left(\mathrm{~d}_{2}{ }^{58}\right)=434 & \mathrm{~F}_{2}{ }^{2}\left(\mathrm{~d}_{2}{ }^{58}\right)=64
\end{array}
$$

Stage realization $\mathrm{d}_{2}{ }^{58}$ is dominated, hence we remove it from further considerations. We select $\mathrm{d}_{2}{ }^{51}$ as the final realization for Stage 2 .

Step 14. Using the transfer function, we determine the final state of the process. Thus, we have:

$$
\Omega_{2}\left(\mathrm{y}_{2}{ }^{(5)}, \mathrm{x}_{2}{ }^{(1)}\right)=\mathrm{y}_{3}{ }^{(1)} .
$$

$\underline{\text { Step } 15 .}$ We set $t=3$ where $t>2$.

Step 16. We add the satisfactory realization generated to the set of potential realizations: $\mathrm{D}^{\mathrm{P}}=\left\{\mathrm{d}^{151}\right\}$ 


\section{Selection of the final realization}

Step 17. Using the algorithm for testing efficiency, we determine that realization $\mathrm{d}^{151}$ is not efficient; there exists one efficient realization which is better, namely $\mathrm{d}^{315}$.

Step 18. We add realization $\mathrm{d}^{315}$ to the set of potential realizations:

$$
\mathrm{D}^{\mathrm{P}}=\mathrm{D}^{\mathrm{P}} \cup\left\{\mathrm{d}^{315}\right\}=\left\{\mathrm{d}^{151}, \mathrm{~d}^{315}\right\}
$$

Step 19. The decision maker does not want to repeat the procedure to obtain another satisfactory realization. We proceed to Step 20.

$\underline{\text { Step } 20 .}$. The decision maker analyzes the set of potential realizations. The values of the multistage criteria for the satisfactory realization $\mathrm{d}^{151}$ are as follows:

$$
\mathrm{F}^{1}\left(\mathrm{~d}^{151}\right)=987 \quad \mathrm{~F}^{2}\left(\mathrm{~d}^{151}\right)=132
$$

The values of multistage criteria for the efficient realization $\mathrm{d}^{315}$ which is better than the satisfactory realization $\mathrm{d}^{151}$ are as follows:

$$
\mathrm{F}^{1}\left(\mathrm{~d}^{315}\right)=987 \quad \mathrm{~F}^{2}\left(\mathrm{~d}^{315}\right)=134
$$

A comparison of the multistage values for both realizations indicates that the only difference occurs in the case of criterion $\mathrm{F}^{2}$. The generated satisfactory realization has a value which is smaller by $1.5 \%$ than the achievable value of this criterion for realization $\mathrm{d}^{315}$.

A list of stage values for both realizations, including maximal stage values (which are not achievable within a single realization) is shown in Table 3 . The emphasized values are those obtained using criteria $\mathrm{F}_{1}{ }^{1}$ and $\mathrm{F}_{2}{ }^{2}$, that is, the most important stage criteria in Stages 1 and 2, respectively.

\begin{tabular}{|l|c|c|c|c|}
\hline \multicolumn{1}{|c|}{ Description } & $\mathrm{F}_{1}{ }^{1}$ & $\mathrm{~F}_{1}{ }^{2}$ & $\mathrm{~F}_{2}{ }^{1}$ & $\mathrm{~F}_{2}{ }^{2}$ \\
\hline Maximal stage values & 499 & 69 & 499 & 69 \\
\hline Satisfactory realization $\mathrm{d}^{151}$ & 498 & 67 & 489 & 65 \\
\hline Better efficient realization $\mathrm{d}^{315}$ & 489 & 67 & 498 & 67 \\
\hline
\end{tabular}

Table 3: Values of stage criteria

We will compare these values. Selecting the efficient realization $\mathrm{d}^{315}$ as the final realization leads to a loss of nine units from the value for criterion $\mathrm{F}_{1}{ }^{1}$ (that is, $1.81 \%$ ) which can be achieved when the satisfactory realization $\mathrm{d}^{151}$ is selected. Selecting the efficient realization $\mathrm{d}^{315}$, in turn, leads to an increase in the value of criterion $\mathrm{F}_{2}{ }^{2}$ by two units (that is, 2.98\%). Consequently, the decision maker selects $\mathrm{d}^{315}$ as the final realization. 


\section{Conclusion}

The present paper is devoted to the problem of selecting the final realization of a multistage multicriteria decision process with the use of stage values. We have considered a situation with a stage-dependent changeable hierarchy of stage criteria. For making the final decision, the paper proposes that the decision consider both the values of stage criteria and those of multistage criteria. The satisfactory realization generated in this procedure need not be efficient, but may generate a set of better efficient realizations, among which one can search for a realization most similar to the satisfactory realization.

In the paper, we have formulated proposals for selecting both the initial state of the process and satisfactory stage decisions. In the illustrative example, we have presented a method of analyzing the set of potential decisions. The most important aspect of this analysis is the decision maker's expert knowledge. Formalizing the process to select the final realization from the set of potential realizations is an open problem.

\section{Acknowledgement}

This research was supported by the National Science Center, decision no. DEC2013/11/B/HS4/01471

\section{References}

[1] Brotcorne, L., Hanafi, S. and Mansi, R. (2013). One-level reformulation of the bilevel knapsack problem using dynamic programming. Discrete Optimization, 10(1), 1-10.

[2] Gulek, M. and Toroslu, I. H. (2010). A dynamic programming algorithm for tree-like weighted set packing problem. Information Sciences, 180(20), 39743979.

[3] Hermann, S. and Klette, R. (2012). Hierarchical scan-line dynamic programming for optical flow using semi-global matching. In Asian Conference on Computer Vision , 556-567. Springer Berlin Heidelberg.

[4] Hsu, W. T., Holmes, T. J. and Morgan, F. (2014). Optimal city hierarchy: a dynamic programming approach to central place theory. Journal of Economic Theory, 154, 245-273.

[5] Miller, C. W. and Yang, I. (2015). Optimal dynamic contracts for a large-scale principal-agent hierarchy: a concavity-preserving approach. arXiv preprint arXiv:1506.05497.

[6] Romano, F., Del Prete, A., Mansard, N., and Nori, F. (2015). Prioritized optimal control: a hierarchical differential dynamic programming approach. 
In 2015 IEEE International Conference on Robotics and Automation (ICRA), 3590-3595. IEEE.

[7] Shen, C. C., and Chen, Y. L. (2008). A dynamic-programming algorithm for hierarchical discretization of continuous attributes. European Journal of Operational Research, 184(2), 636-651.

[8] Steuer, R.E. (1986) Multiple criteria optimization theory: Computation and Application, J.Willey, New York.

[9] Trzaskalik, T. (2009). Changeable, weighted importance of criteria in multiobjective dynamic programming. In: J.Biolik (ed): Econometrics Dilemmas. The Karol Adamiecki University of Economics in Katowice Press. Katowice (in Polish).

[10] Trzaskalik, T. (1998). Multiobjective analysis in dynamic environment. The Karol Adamiecki University of Economics in Katowice Press.

[11] Trzaskalik, T. (1998). Single and group hierarchy in multiple objective dynamic programming. In: Preis de décision multicritére: raison d'être en gestion. Cahier de la recherche No 98-1. Université du Québek an Abitibi-Temiscamingue.

[12] Trzaskalik, T. (1997). Hierarchy depending on state in multiple objective dynamic programming. Operations Research and Decisions no 2, 65-73.

[13] Trzaskalik T. (1995) Hierarchy depending on value in multiple criteria dynamic programming. Foundations on Computing and Decision Science, 20, 2, 139-148.

[14] Trzaskalik, T. (1992). Hierarchical approach to multi-criteria dynamic programming. Information Systems and Operational Research INFOR, .30, 2, 132142.

[15] Trzaskalik, T. (1990). Multiobjective discrete dynamic programming. Theory and applications in economics. The Karol Adamiecki University of Economics in Katowice Press, Katowice, 1990 (in Polish)

[16] Van Meerbergen, G., Vergauwen, M., Pollefeys, M., and Van Gool, L. (2002). A hierarchical symmetric stereo algorithm using dynamic programming. International Journal of Computer Vision, 47(1-3), 275-285. 